\title{
Brève notice d'histoire littéraire : la question de la rhétoricité sévignéenne
}

\section{Christine Noille}

\section{(2) OpenEdition \\ 1 Journals}

\section{Édition électronique}

URL : http://journals.openedition.org/rhetorique/442

DOI : $10.4000 /$ rhetorique.442

ISSN : 2270-6909

\section{Éditeur}

UGA Éditions/Université Grenoble Alpes

\section{Édition imprimée}

ISBN : 978-2-84310-316-2

\section{Référence électronique}

Christine Noille, «Brève notice d'histoire littéraire : la question de la rhétoricité sévignéenne », Exercices de rhétorique [En ligne], 6 | 2016, mis en ligne le 08 février 2016, consulté le 12 septembre 2020. URL: http://journals.openedition.org/rhetorique/442 ; DOI : https://doi.org/10.4000/rhetorique.442

Ce document a été généré automatiquement le 12 septembre 2020.

\section{(c) (i) (9)}

Les contenus de la revue Exercices de rhétorique sont mis à disposition selon les termes de la Licence Creative Commons Attribution - Pas d'Utilisation Commerciale - Partage dans les Mêmes Conditions 4.0 International. 


\title{
Brève notice d'histoire littéraire : la question de la rhétoricité sévignéenne
}

\author{
Christine Noille
}

1 Une question est souvent posée dès qu'on commence à travailler sur l'idée d'une rhétoricité de Sévigné : Sévigné connaissait-elle la rhétorique ? Sévigné écrivait-elle en rhétoricienne ? Poser quelques jalons en guise de réponse est le but principal de cette notice.

2 La rhétorique est habituellement définie comme un art de parler (ou un art de persuader, ou un art d'écrire, c'est selon), mais toujours comme un art à destination auctoriale, un ensemble de préceptes pour les auteurs, une technique leur permettant d'inscrire une forme, un schéma, une figure dans un texte. Et il en va des règles pour bien écrire comme de n'importe quelle technique de métier, rien ne vient garantir que l'auteure Sévigné - une femme, aristocrate - ait acquis un quelconque savoir spéculatif et pratique en matière de rhétorique du discours ni même qu'elle ait songé, le cas échéant, à le mobiliser dans l'écriture épistolaire.

3 Essayons donc de démêler ce qu'on peut établir, de l'ensemble des suppositions et interprétations - étant entendu que l'histoire est toujours peu ou prou une interprétation comme une autre ${ }^{1}$.

\section{Un art de lire en rhétorique?}

4 Madame de Sévigné revendique à plusieurs reprises non pas un art d'écrire, mais un art de lire et de juger l'écriture ou le discours, une science, une expertise de l'éloquence :

Vous vous souvenez peut-être assez de moi pour savoir que je suis blessée des méchants styles ; j'ai quelque lumière pour les bons, et personne n'est plus touchée que moi des charmes de l'éloquence. (12 juillet $1671:$ I, 294²) 
Il s'agit là au demeurant d'une pratique collective, prenant place dans le cadre d'une lecture orale où l'art de juger devient un art de remarquer :

Nous lisons toujours Le Tasse avec plaisir. Je suis assurée que vous le souffririez, si vous étiez en tiers; il y a bien de la différence entre lire un livre toute seule, ou avec des gens qui l'entendent et relèvent les beaux endroits et qui, par là, réveillent l'attention. (15 juillet $1671: \mathrm{I}, 296$ )

6 Savoir repérer les « beautés » dans le flux discursif et savoir les apprécier comme telles relèvent bien alors d'une « science » à part entière, que la Marquise revendique et dont elle va jusqu'à s'enorgueillir :

Nous achevons Le Tasse avec plaisir; nous y trouvons des beautés qu'on ne voit point quand on n'a qu'une demi-science. (12 juillet 1671 : I, 293)

7 Cet art de lire est donc un art de remarquer des formes et des formules remarquables, autrement dit un art du remarquage culturellement médiatisé, dans ce milieu à la fois érudit et mondain, par ce qu'Umberto Eco nommerait la compétence «encyclopédique » du lectorat, en l'occurrence une encyclopédie et une bibliothèque, une encyclopédie des formes remarquables d'une part (que nous interpréterons comme une connaissance des formes remarquées et identifiées par l'analyse rhétorique et poétique pour rendre compte des séquences d'un énoncé, pour les décrire); et d'autre part une bibliothèque, la mémoire d'un corpus massif de textes mémorisés en tant qu'ils exemplifient ces formes remarquables.

8 Bref ce qui se profile ici, c'est l'hypothèse heuristique d'un milieu dans lequel est pertinente l'idée d'un art de lire en connaisseur, partagé par des publics masculins et féminins, qui est tout autant travaillé par l'axiologie du milieu galant que modélisé par le rapport aux textes que l'épistémè rhétorique structure, en particulier par le biais des institutions pédagogiques.

9 Car il existe un art de lire en rhétoricien(ne) qui double, complète et sans doute précède les arts d'écrire.

\section{Lire en professionnel de la rhétorique : les analyses des maîtres}

10 Comment lisent alors, non pas les familiers du cercle de Sévigné, mais précisément les maîtres de rhétorique, ce pôle à la fois extérieur (car pédagogue et professionnel) et solidaire (en tant qu'il porte - à un degré d'expertise technique sans doute renforcée un certain nombre de gestes critiques, de façons de remarquer, de faire attention au texte, qui sont aussi le fait des lecteurs se revendiquant connaisseurs)? Nous renverrons ici à la présentation détaillée des commentaires rhétoriques que nous avons donnée récemment ${ }^{3}$ et nous donnerons simplement ici en parallèle un texte (un bref extrait de l'Énéide) et le commentaire rhétorique du padouan Ferrazzi :

[VIRGILE, Énéide I, 65-75]

65 Éole, lui dit-elle d'un air suppliant, vous que le Père des Dieux a rendu l'arbitre du calme et de l'orage,

67 voyez cette flotte qui vogue sur la mer de Toscane : ce sont mes ennemis : ce sont les Troyens vaincus, qui veulent aborder en Italie et s'y établir.

69 Déchaînez vos vents, submergez 70 ou dispersez ces vaisseaux [et leurs corps en pleine mer].

71 J'ai quatorze Nymphes d'une beauté parfaite ; Déiopée, qui l'emporte sur toutes les autres, sera le prix du service que vous m'aurez rendu. Je vous unirai ensemble 
pour toujours, et les enfants qui naîtront de cet heureux hyménée, seront beaux comme elle ${ }^{4}$.

[FERRAZZI ${ }^{5}$ Junon à Éole, afin qu'il déchaîne ses vents contre la flotte des Troyens.

Émotion : Gratitude.

Attitude : qui convient à une suppliante

Lieux communs de l'argumentation : tirés des Circonstances non négligeables

Deux parties : l'Exorde et l'Exposé des arguments

Éole] Dans l'Exorde, pour se concilier les bonnes dispositions d'Éole, elle exalte son pouvoir sur les flots, puisqu'il peut les apaiser comme les agiter.

Voyez cette flotte] Exposé des arguments, qui consiste apparemment en ce raisonnement :

Tu peux et tu dois m'accorder cette grâce.

Donc tu le feras.

Qu'il le puisse, cela est rendu évident par le pouvoir que lui avait donné Jupiter sur les flots; qu'il le doive, elle le montre en tirant argument de circonstances non négligeables: un peuple vaincu et ennemi de Junon, lui aussi se devait de l'avoir en haine éternelle.

Déchaînez] Conclusion de l'Enthymème, conclusion qui formule la Requête ellemême.

J'ai quatorze nymphes] Pour qu'il obéisse plus vite à la requête de Junon, elle lui expose la faveur qui en découlera, à savoir l'union avec Déiopée ; et elle renforce cette faveur en tirant argument de circonstances non négligeables: elle est la plus belle des Nymphes, jamais elle ne rompra ce mariage ; et en même temps en tirant argument de l'espoir d'une descendance ; Arist., II, 7, 80.

Comment résumer et caractériser cet art de lire en rhétoricien? Des lignes de force apparaissent ici visiblement.

- Il s'agit d'abord d'un art de diviser le discours en séquences remarquables et de décrire les séquences en les identifiant à l'aide de cette encyclopédie des formes simples qu'a constituée la rhétorique - principalement, avec les maîtres de rhétorique, l'encyclopédie des ressources argumentatives et des lieux du pathos, très accessoirement l'encyclopédie des figures de l'éloquence.

- Il s'agit également d'un art de modéliser la cohérence globale des séquences sur des canevas dispositionnels types, ceux des genres de discours (que la tradition rhétorique ne cesse, là aussi, d'affiner et de compléter) ; et à partir de là d'identifier le genre du discours opératoire (ici en l'occurrence, une exemplification exemplaire et pour tout dire célébrissime de la petitio ou requête $\left.{ }^{6}\right)$.

- Il s'agit enfin d'un art d'évaluer la conduite, la composition, la gestion dynamique de la dispositio en tant qu'elle est finalisée sur un dessein probatoire; un art de remarquer la négociation de l'enchaînement et de la transition des séquences : bref, un art d'apprécier le déroulé discursif, la conduite, ou encore, pour reprendre le terme rhétorique technique et donc latin, le ductus?

12 Si l'on en revient alors aux connaissances de Sévigné et à sa pratique d'une lecture éclairée de "lumières » en matière d'éloquence, qu'en est-il de son expertise en comparaison de celle des maitres de rhétorique?

Pour aller vite, il est aisé de montrer qu'au fil de ses remarques sur des lectures ou des auditions, Sévigné met en avant des compétences dans les trois compartiments que nous venons de rappeler: elle manifeste un art de remarquer et d'identifier formellement des séquences, ainsi qu'une sensibilité au ductus, à la conduite, au mouvement du discours; mais également, de façon tout à fait notable durant la grande 
période de lectures de l'été 1671 , un art de modéliser les textes lus selon des canevastype.

\section{Plus « qu'une demi-science »: les savoirs techniques de Sévigné}

\subsection{Sévigné rhétoricienne}

Un rapide relevé de quelques énoncés ostensiblement informés par la rhétorique et la poétique suffira.

Qu'y a-t-il de remarquable dans un discours? En premier, le ductus, sa conduite au sens rhétorique du terme, c'est-à-dire la dynamique dispositionnelle qui entraîne l'ordre d'un argumentaire comme celui d'un récit. Voici d'abord des remarques sur l'écriture tacitéenne de l'histoire, focalisées exclusivement sur la question du ductus dans son lien avec l'intérêt du récit (nous mettons en italiques le lexique de la conduite) :

Auriez-vous été assez cruelle pour laisser Germanicus au milieu de ses conquêtes et dans ces marais d'Allemagne, sans lui donner la main pour l'en tirer? Ne voulezvous point au moins le conduire jusqu'au festin où il fut empoisonné par Pison et sa femme ? [....] Mon fils, comme je vous ai dit, m'a fichée dans le milieu de Cléopâtre, et je l'achève; cela est d'une folie dont je vous demande le secret. J'achève tous les livres, et vous les commencez. (8 juillet $1671:$ I, 291)

Et voici, surtout, l'analyse rhétorique d'une oraison funèbre subordonnée à sa conduite (nous donnons entre crochets les termes techniques latins auxquels réfèrent les expressions françaises) :

Il est venu un jeune père de l'Oratoire pour faire l'oraison funèbre. [...] Mais en sortant de son trouble, il est entré dans un chemin [ductus] si lumineux; il a si bien établi [ressources de l'inventio] son discours; il a donné au défunt des louanges si mesurées [laudatio: première partie de l'oratio funebris]; il a passé dans tous les endroits délicats [loci laudateurs difficiles à abonder] avec tant d'adresse; il a si bien mis dans son jour [amplificatio] tout ce qui pouvait être admiré ; il a fait des traits d'éloquence et des coups de maitre [figurae et sententiae] si à propos et de si bonne grâce, que tout le monde, je dis tout le monde, sans exception, s'en est écrié, et chacun était charmé d'une action [actio, "discours public ${ }^{8}$ "] si parfaite et si achevée. (6 mai $1672:$ I, 503)

Nous avons sans doute là l'un des exemples les plus probants en faveur d'une auteure experte en rhétorique de la lecture, capable non seulement d'identifier le "pattern » général (l'oraison funèbre) et ses passages obligés (l'éloge, l'amplification), mais encore de délimiter des séquences (« les endroits délicats »), de les mettre en réseau (« dans un chemin si lumineux ») et de remarquer les formules (" traits », " coups de maittre ») qui sortent de l'ordinaire'.

Rien d'étonnant alors à ce que ses lettres abondent en descriptions métatextuelles précisément informées par les catégories de la rhétorique, telles que la division du sermon en trois points ${ }^{10}$, la digression ${ }^{11}$ ou encore les stéréotypies de la péroraison ${ }^{12}$.

\subsection{Sévigné poéticienne}

Mais la lecture de Sévigné a également recours à des formes-types débattues et définies dans la poétique. C'est ainsi qu'elle est capable de parler d'une histoire en termes de 
poétique théâtrale, c'est-à-dire de sujet, d'intrigue, de règle, d'émotion et de personnage :

C'est le juste sujet d'une tragédie dans toutes les règles du théâtre. Nous en réglions les actes et les scènes l'autre jour; nous prenions quatre jours au lieu de vingtquatre heures, et c'était une pièce parfaite. Jamais il ne s'est vu de si grands changements en si peu de temps, jamais nous n'avez vu une émotion si générale; jamais vous n'avez ouï une si extraordinaire nouvelle. M. de Lauzun a joué son personnage en perfection. Il a soutenu son malheur avec une fermeté, un courage, et pour tant un douleur mêlée d'un profond respect, qui l'ont fait admirer de tout le monde. [...] Mademoiselle a fort bien fait aussi. (À Coulanges, 24 décembre $1670:$ I, 143-144)

Où l'on voit, en passant, que non seulement Madame de Sévigné suit l'actualité théâtrale (convocation du modèle cornélien de la fermeté dans la douleur et du courage admirable pour mettre en scène le personnage de Lauzun) mais qu'elle possède une connaissance positive des règles du poème dramatique (et en particulier de la tragédie, même s'il lui arrive aussi d'évoquer la comédie ${ }^{13}$ ).

Elle avoue une égale adresse dans l'art d'analyser le récit en prose (que ce soit le récit des nouvelles du jour ou les récits fictifs des romans). C'est ainsi qu'il lui arrive de faire référence au dispositif romanesque du début in medias res et de son corollaire, l'analepse explicative :

Ce qui s'appelle tomber du haut des nues, c'est ce qui arriva hier au soir aux Tuileries; mais il faut reprendre les choses de plus loin. Vous en êtes à la joie, aux transports, aux ravissements de la princesse et de son bienheureux amant [...]. ( Coulanges, 19 décembre $1670:$ I, 140-141)

Et de même qu'elle analyse selon des marqueurs rhétoriques l'oraison funèbre à laquelle elle a assistée, elle est également capable de parler de la lecture d'un roman en termes de style, de sentiments, de passions, d'action, de dénouement et encore une fois, de conduite - autant de termes dont il serait inutile de démontrer la technicité au XVII ${ }^{\mathrm{e}}$ siècle tant la chose est aujourd'hui établie ${ }^{14}$ :

Je songe quelquefois d'où vient la folie que j'ai pour ces sottises-là ; j'ai peine à le comprendre. [...] Le style de la Calprenède est maudit en mille endroits : de grandes périodes de roman, de méchants mots, je sens tout cela. J'écrivis l'autre jour une lettre à mon fils de ce style, qui était fort plaisante. Je trouve donc qu'il est détestable, et je ne laisse pas de m'y prendre comme à de la glu. La beauté des sentiments, la violence des passions, la grandeur des événements, et le succès miraculeux de leur redoutable épée, tout cela m'entraîne comme une petite fille; j'entre dans leurs desseins. (12 juillet 1671 : I, 294. Nous mettons en italiques les termes techniques.)

Ce sont bien les catégories aristotéliciennes qui sont à l'arrière-plan doctrinal de ce commentaire informé que n'auraient pas renié un Valincour ou un Du Plaisir et qui sont ici utilisées dans ce qui semble être une nouvelle variation mondaine sur la définition du roman en marge de la définition canonique de la tragédie au chapitre VI de la Poétique. \\ 3.3. Sévigné juge de Sévigné : le cas de la narratio}

$\mathrm{Au}$ croisement de la rhétorique et de la poétique enfin, l'on trouve toute une série de remarques sur la conduite de la narratio probatoire (récit à vocation persuasive dans un dispositif argumenté) dans l'écriture épistolaire, lesquelles ne peuvent se comprendre qu'en référence à la proposition définitoire du De Inventione : 
Elle [la narration] a le mérite de la brièveté, si l'orateur commence où il faut commencer, sans remonter trop haut [non ab ultimo repetetur]; s'il ne donne point des détails, quand il ne faut que des résultats ; car souvent il suffit d'énoncer un fait sans en développer les circonstances; s'il s'arrête au moment de dire des choses inutiles ; s'il ne s'égare pas dans des digressions ; [...] enfin s'il ne se répète jamais, s'il ne revient jamais sur ses pas ${ }^{15}$.

Autrement dit, le mot d'ordre de la narratio (en particulier judiciaire) est l'utilité des circonstances choisies :

La narration n'est pas dans un jour favorable, [...] quand elle est obscure et négligée dans ce qui nous est avantageux. Pour éviter cet écueil, ramenez tout à l'intérêt de votre cause; supprimez, autant qu'il est possible, toutes les circonstances défavorables; glissez légèrement sur tout ce qui est dans l'intérêt de votre adversaire ; mais développez avec soin, avec clarté tout ce qui peut vous servir ${ }^{16}$.

Tel est donc le contexte rhétorique de la technique narrative, que nous sommes bien obligés de restituer au lecteur contemporain, de façon à montrer comment non seulement Madame de Sévigné y réfère, mais même en joue.

Référence au dispositif persuasif standard de la narration tout d'abord, dont l'épistolière moque discrètement la lourdeur et la longueur :

Voici comme la chose se passa [...]. Voilà une belle narration bien divertissante et bien nécessaire, mais elle est vraie, ma bonne. Il n'y a pas un mot pour un autre, et j'admire qu'il [M. d'Acqueville] vous ait voulu mander cette bagatelle d'une façon si contraire à la vérité. Vous pouvez croire que voilà la dernière fois que j'en parlerai, mais j'ai voulu vous dire la chose tout juste et tout naïvement comme elle s'est passée, et vous faire voir que si j'avais été d'abord en état de songer à quelqu'un, j'aurais songé à lui [...]. Si vous n'êtes fatiguée de ce récit, vous avez une bonne santé ; je fais vœu de n'en faire jamais un si long. (7 juin 1671 : I, 264-265)

Et référence ironique, célèbre entre toutes, au dispositif perverti de la narration circonstanciée superfétatoire dans la lettre des foins :

[...] et comme il est frère du laquais de Mme de Coulanges, je suis bien aise de vous rendre compte de mon procédé. [...] Voilà l'histoire en peu de mots. Pour moi, j'aime les narrations où l'on ne dit que ce qui est nécessaire, où l'on ne s'écarte point à droite, ni à gauche, où l'on ne reprend point les choses de si loin. (À Coulanges, 22 juillet $1671: \mathrm{I}, 303-304)$

Disons pour résumer que Madame de Sévigné possède plus de connaissances techniques que la plupart d'entre nous : car non seulement elle maitrise leurs références, présentes à son esprit au moment où elle émet un commentaire, mais elle est capable de les appliquer avec pertinence à la description d'énoncés. Il s'agit là bien "plus qu'une demi-science » : un savoir théorique et son usus, sa mise en œuvre dans une pratique, qui certes n'est pas d'écriture, mais de lecture.

\section{En guise de bilan. De la lecture à l'écriture : le saut herméneutique}

31 Ce que nous avons essayé ici de mettre en place, c'est la légitimité contextuelle qu'il y a à ancrer le corpus des lettres sévignéennes dans une pratique lectoriale rhétoricienne. C'est ainsi que nous avons répertorié un certain nombre de cas où Mme de Sévigné commente avec les outils de la rhétorique et de la poétique un certain nombre de textes, ceux d'autrui comme ses propres lettres. Nous nous permettons également de renvoyer à une de nos précédentes analyses concernant une autre question, tout à fait 
décisive en rhétorique, le débat qui se poursuit en 1671 et 1672 entre Mme de Sévigné et Mme de Grignan concernant la dispositio, la composition, dans l'extension et la tenue des longues lettres, ce que nous avons appelé l'enjeu de la « lisibilitée ${ }^{17} »$ : ne pas perdre le ductus, le fil, sous l'effet de la division, de l'éparpillement en de multiples nouvelles et de l'absence de mobilisation d'un canevas global de cohérence. Il s'agit là, pour les deux interlocutrices, d'un enjeu cognitif et esthétique majeur, puisqu'il touche à la fois à l'intelligibilité de la lettre, à sa mémorisation et au plaisir de la lecture suivie.

Mais, et c'est là un tournant majeur dans cette présente notice, il n'en reste pas moins qu'il y a une rupture épistémologique entre acter une pratique lectoriale rhétoricienne chez Mme de Sévigné lectrice et commentatrice (y compris de son propre texte) et repérer systématiquement dans son écriture des éléments rhétoriques ou poétiques embrayeurs de structuration. D'un côté, nous avons attesté une pratique sévignéenne de lecture; de l'autre côté, c'est en revanche notre propre analyse aujourd'hui, c'est notre description qui est en jeu, c'est notre pratique d'une lecture rhétoricienne, d'un art de marquer et de remarquer dans le texte des dispositifs rhétoriques embrayeurs de lisibilité et d'efficacité, en rapport avec une encyclopédie actualisée des formes et des figures.

Entre les deux, le point obscur, c'est bien évidemment la pratique d'écriture de Sévigné : Sévigné lit en rhétoricienne, je la lis en néo-rhétoricienne, mais écrivait-elle en rhétoricienne ? Écrit-elle sciemment selon une bibliothèque et une encyclopédie des formes et figures de la rhétorique ? Y a-t-il de sa part une inscription auctoriale de dispositifs rhétoriques? Ou bien leur rencontre, leur convocation, si tant est qu'elles sont avérées, sont-elles le produit d'un habitus aristocratique et d'une culture galante, le fruit non pas du judicium, mais de l'usus et de l'ingenium ? Elle qui lit en connaisseur, écrit-elle en connaissance de cause, ou simplement d'un jet, comme elle l'affiche et l'exhibe, tout au plus en première lectrice de ses lettres?

Nous dirons volontiers que la réponse à cette question est à la fois capitalissime et négligeable. Capitalissime du point de vue de l'idéologie historique (idéologie de l'auteur, de son projet, du statut de la création, etc.), c'est-à-dire du point de vue d'une herméneutique de l'intentionnalité et d'une herméneutique de l'écriture. Mais la réponse à cette question est à peu près négligeable du point de vue qui intéresse le rhétoricien, à savoir le texte, sa cartographie, sa description, son intelligibilité, son efficacité.

En effet, vouloir établir une relation de cause à effet entre la lecture rhétorique de Madame de Sévigné et une pratique rhétoricienne de l'écriture est d'un certain point de vue strictement accessoire : c'est un problème qui ne change rien à la matérialité du texte donné à lire et aux lectures rhétoriciennes qui peuvent en être faites, hier comme aujourd'hui, et qui remarquent en lui des dispositifs remarquables. Ont-ils été intentionnellement prescrits? Sont-ils le fait d'un habitus ? Ou sont-ils la projection de la culture du lecteur?

Si l'on répond par l'affirmative à l'une ou l'autre des deux premières questions, alors on commet un double écart : on s'engage sur une interprétation de l'auctorialité (dans le sens d'un artifice, d'une maitrise empirique, d'un naturel, d'un génie, etc.) et on établit la valeur de la description que l'on voudrait faire d'un texte sur l'image auctoriale ainsi construite. Double imposture méthodologique, puisque le premier geste relève d'un pari idéologique, et le second d'une erreur épistémologique : la validité d'une analyse ne dépend pas de sa vraisemblance historique. Toute analyse ne connait qu'une seule 
actualité, celle du présent du commentateur. Et sa validité dépend à la fois de la correction avec laquelle elle est effectuée et de sa compatibilité avec le fait du texte.

Ou pour le dire autrement, les dispositifs rhétoriques ou poétiques embrayeurs d'effets sont quelque part dans le texte, mais ils sont rarement des données objectives incontournables (même à supposer qu'un auteur sur-inscrive et en quelque sorte exhibe un certain nombre de dispositifs embrayeurs d'organisation, ce ne sont pas les seuls, ni les plus efficaces peut-être, qui sont susceptibles d'être investis par le lecteur). La visibilité, la "remarquabilité » de tels dispositifs dépend bel et bien d'abord du lecteur, de son aptitude à les remarquer, autrement dit de la bibliothèque et de l'encyclopédie des formes et des figures dont il dispose, de son expertise à la fois spéculative et technique en matière de rhétorique.

Que les premiers lecteurs de Mme de Sévigné, un La Rochefoucauld, un Bussy-Rabutin, un Coulanges, un Pomponne, disposaient de cette compétence-là, rien de plus évident ; que Mme de Sévigné et son entourage proche (au premier rang duquel son fils, le frater, qui écrit remarquablement, soit dit en passant, voir sa lettre du 6 mars $1671:$ I, 178) en disposaient également, nous l'avons établi. Mais il en va des formes de la rhétorique comme d'autres outils de la critique contemporaine : la pertinence de leur mobilisation sur un texte donné dépend d'abord de la compétence du relecteur. Et à moins d'imaginer un nouveau Pierre Ménard ayant la prétention de lire les lettres de Madame de Sévigné comme Madame de Sévigné, on est devant deux tâches complémentaires mais distinctes qu'en son temps Barthes a fort bien décrites ${ }^{18}$ : étudier les institutions culturelles de production et de réception des écrits; et étudier la configuration singulière d'énoncés qui ont pris place dans la série des productions culturelles à une époque donnée et n'en finissent pas de prendre place dans d'autres séries de réception à diverses époques.

Reste donc à laisser Sévigné aux historiens de la fonction « auteur » et le statut de la lettre aux historiens des genres épistolaires et des catégories rhétoriques. Et revenons dans notre $\mathrm{XXI}^{\mathrm{e}}$ siècle, pour y interroger notre compétence à relire le texte de Sévigné, à l'analyser, par exemple en manipulant les outils de la rhétorique, de notre rhétorique : qu'ils soient informés par l'archéologie de la discipline ou par la critique du champ actuel. Et dans un cas comme dans l'autre, les modèles et les "patterns » rhétoriques que nous convoquerons pour en tester l'application ou la reformulation dans le syntagme textuel feront également office d'«interprétants", de scénarios herméneutiques dont la mise en situation dans le texte aura permis d'en produire une analyse plus informée et partant littéralement plus «informante », une description « diagnostic » et parfois « pronostic ${ }^{19}$ ».

Pour ce faire, nous avons assurément besoin non seulement d'une théorie rhétorique, mais d'une théorie tournée vers la pratique de lecture. Voilà qui nous invite à plaider pour ce que nous pourrions appeler un tournant cognitif de la rhétorique, qui nous fasse passer d'un énoncé tel que "La rhétorique prend en charge les effets de discours, son efficacité ", à un énoncé tel que "La rhétorique prend en charge les opérations de lecture, de perception et d'intelligibilité des formes discursives »; et plus encore pour un tournant technique de la rhétorique de la lecture, qui nous fasse passer d'un énoncé tel que «La rhétorique prend en charge la compréhension des processus de mise en forme du texte dans la lecture » à un énoncé tel que «La rhétorique prend en charge une méthodologie de leur mise en œuvre par le lecteur ». 
41 Ainsi comprise, la démarche rhétorique remontera des effets aux énoncés, subordonnant sa pertinence à la justesse avec laquelle elle mobilisera les outils d'analyse - et abandonnant ainsi toute prétention idéologique à ancrer sa valeur sur ce qui semble bel et bien devoir être tout juste un "vraisemblable historique", la correspondance qu'il y aurait entre le texte du XVII ${ }^{e}$ siècle et des outils d'analyse qui ont pu lui être contemporains mais dont les usages anciens sont ressaisis, filtrés et interprétés au présent. Bref, plus que jamais «la première règle objective est ici d'annoncer le système de lecture, étant entendu qu'il n'en existe pas de neutre ${ }^{20}$. »

Quant aux quelques données d'histoire littéraire que nous avons rassemblées dans cette notice, que les historiens les réinterprètent et leur donnent sens à la lumière de leurs problématiques.

\section{NOTES}

1. Voir sur ce sujet par exemple M. Charles, L'Arbre et la source, Paris, Seuil, 1984, p. 15 : «Quand l'histoire littéraire ne veut connaître que la date de la production, elle devient une herméneutique comme les autres, décidant que le sens, c'est l'origine. »

2. Données entre parenthèses, les références aux lettres de Mme de Sévigné mentionnent la date de la lettre et sa pagination (tome et page) dans l'édition de référence : Correspondance, éd. R. Duchêne, Paris, Gallimard, coll. "Bibliothèque de la Pléiade ", 1972-1978, 3 tomes. Le destinataire n'est précisé que lorsqu'il ne s'agit pas de Mme de Grignan.

3. Ch. Noille, «Le discours d'un Prince (Énéide I, 597-610) : archéologie de la disposition », dans Exercices de rhétorique 2|2013 (http://rhetorique.revues.org/176).

4. Virgile, Énéide I, 65-75, trad. Desfontaines (1743).

5. M. A. Ferrazzi, Exercitationes Rhetoricae in praecipuas P. Virgilii Maronis orationes, quae in Aeneidum libris leguntur [Exercices de rhétorique sur les principaux discours de Virgile, que l'on trouve dans l'Enéide], Presses du Séminaire [Manfrè], Padoue, 1694, extrait traduit par Ch. Noille en collaboration avec l'E.A. RARE - Rhétorique de l'Antiquité à la Révolution.

6. Sur les genres du discours, voir F. Goyet, «Le problème de la typologie des discours ", dans Exercices de rhétorique 1|2013 (http://rhetorique.revues.org/122); et Ch. Noille, «Les genres du discours dans l'ancienne rhétorique: listes, schémas et mode d'emploi, avec un exemple (le discours de Germanicus) », Exercices de rhétorique 3 | 2014 (http://rhetorique.revues.org/337).

7. Sur cette notion, voir D. Knop, La Cryptique chez Montaigne, doctorat soutenu le 08 déc. 2012 à l'université Grenoble Alpes.

8. Il ne s'agit pas ici de la seule partie de l'actio oratoire (ou déclamation) mais du sens plus général d'actio en latin. Voir E. Littré, Dictionnaire de la langue française, Paris, 1873-1877, s.v. action : «10 $10^{\mathrm{e}}$. Discours public, tel qu'un sermon, une harangue, un plaidoyer. Vieux en ce sens. [...] “[Le prédicateur] a si bien mis dans tout son jour [...] chacun était charmé d'une action si parfaite et si achevée", Sév. 202.»

9. Divisio, ordo, transitio: sur ces trois gestes fondamentaux pour penser la rhétorique de la dispositio (c'est-à-dire une théorie de la composition), voir Ch. Noille, "Les Lettres de Sévigné sont-elles informes? Éléments pour une rhétorique de la disposition », dans C. Lignereux dir., Lectures de Madame de Sévigné. Les lettres de 1671, Presses Universitaires de Rennes, 2012, p. 31-32. 
10. «Je ne vous puis dire combien je vous plains, combien je vous loue, combien je vous admire ; voilà mon discours divisé en trois points. Je vous plains [...]. Je vous loue [...]. Et je vous admire [...]. » (4 mai $1672:$ I, 499).

11. "Nous avons commencé la Morale; c'est de la même étoffe que Pascal. A propos de Pascal, je suis en fantaisie d'admirer l'honnêteté de ces messieurs les postillons [...]. Voilà une belle digression. Je reviens à nos lectures. » (12 juillet $1671:$ I, 293-294).

12. "Adieu, ma chère enfant; je suis à vous, sans aucune exagération ni fin de lettre, hasta la muerte inclusivement. " (4 novembre $1671: \mathrm{I}, 376)$.

13. «Mais sur les sept heures du soir, Sa Majesté étant persuadée, par la Reine, Monsieur et plusieurs barbons [...]. » (à Coulanges, 19 décembre $1670:$ I, 141).

14. Voir en particulier les travaux et les éditions de C. Esmein : en particulier L'essor du roman. Discours théorique et constitution d'un genre littéraire au XVII siècle, Paris, H. Champion, 2008 ; et Poétiques du roman. Scudéry, Huet, Du Plaisir et autres textes théoriques et critiques du XVII siècle sur le genre romanesque, Paris, H. Champion, 2004.

15. Cicéron, De Inventione, I, XX, 28, trad. Nisard, 1869.

16. Ibid. (XXI, 30). Sur la narratio, voir Ch. Noille, "L'intelligence du récit. Enquête dans la pédagogie rhétorique des dispositifs narratifs ", dans La Lettre de l'AIRDF, $\mathrm{n}^{\circ}$ 57, "L'exercice en examen ", coord. C. Ronveaux, 2015.

17. Voir Ch. Noille, «Les Lettres de Sévigné sont-elles informes? Éléments pour une rhétorique de la disposition », dans C. Lignereux dir., Lectures de Madame de Sévigné. Les lettres de 1671, Presses Universitaires de Rennes, 2012, p. 19-47.

18. R. Barthes, Sur Racine, Paris, Seuil, 1963 ; rééd. dans CEuvres complètes II, Paris, Seuil, 2002, «Histoire et littérature : à propos de Racine » (initialement paru dans Annales. Économie, Société, Civilisations, 1960, vol. 15, $\mathrm{n}^{\circ}$ 3, p. 524-537).

19. Nous reprenons ici les termes que Raphaël Baroni a familiarisés pour décrire les opérations de modélisation du syntagme du récit en fonction de scénarios type que le lecteur compétent mobilise dans le fil de sa lecture pour pronostiquer l'issue possible ou diagnostiquer l'histoire sous-jacente à l'œuvre. Voir R. Baroni, La Tension narrative. Suspense, curiosité et surprise, Paris, Seuil, 2007.

20. Ibid., p. 194.

\section{AUTEUR}

\section{CHRISTINE NOILLE}

Université Grenoble Alpes - UMR Litt\&Arts / RARE Rhétorique de l'Antiquité à la Révolution 